\section{Endoscopic and Percutaneous Drainage of Symptomatic Walled-Off Pancreatic Necrosis Reduces Hospital Stay and Radiographic Resources}

\author{
Michael Gluck, Andrew Ross, Shayan Irani, \\ Otto Lin, Ellen Hauptmann, Justin Siegal, \\ Mehran Fotoohi, Robert Crane, David Robinson, \\ Richard A. Kozarek
}

Clin Gastroenterol Hepatol 2010;8:1083-88

Walled off pancreatic necrosis (WOPN), a potentially serious complication of acute severe pancreatitis, has been conventionally managed surgically. Advances in interventional radiology and endoscopy have resulted in development of minimally invasive interventional radiological and endoscopic techniques for management of WOPN. The experience with these new techniques is limited but encouraging. These techniques involve multiple procedures and imaging techniques that adds on to the cost of the treatment. The authors of the current study had earlier described the technical aspects of combined modality therapy (CMT) for patients with symptomatic WOPN that involved combination of percutaneous large-bore catheter drainage with endoscopic transgastric or transduodenal stent placement into the necrotic fluid collection. [1] In the current study the authors retrospectively compared the outcome of CMT (23 patients) with that of standard percutaneous drainage (SPD) alone (43 patients) in patients with symptomatic WOPN.

In patients with SPD group, radiologically guided percutaneous drainage of WOPN was done with aspiration of as much fluid as possible at the time of the drainage. This was followed by placement of $12 \mathrm{~F}$ drainage tubes that were irrigated with $10-20 \mathrm{~mL}$ of sterile saline 3 times daily. Percutaneous catheters were sequentially upsized to a maximum of 28F. In the CMT group, initially computed tomography (CT) guided drains were placed into the WOPN but in contrast to the SPD group, only $10 \mathrm{ml}$ of the fluid was aspirated and then the drains were clamped. The patients then immediately underwent endoscopic transgastric or transduodenal drainage of the WOPN. Endoscopic ultrasound (EUS) was used when there was no definable luminal bulge. Endoscopic retrograde cholangiopancreatography (ERCP) was performed and if leak was present pancreatic duct stent was inserted. Biliary stent was placed in patients with biliary obstruction. A nasojejunal feeding tube was placed in all the patients. Antibiotics were given as per the culture sensitivity reports. Percutaneous drain tubes were removed when there was no fluid draining from them, clamping them did not cause symptoms and there was no residual collection on CT scan. Endoscopically placed transmural stents were removed if there was no pancreatic duct disruption, the fluid collection had resolved, and removal of percutaneous catheter did not result in fluid reaccumulation. In patients with disconnected pancreatic duct, transenteric stents were left in place indefinitely.

Forty-three patients (25 males) underwent SPD and 23 (18 males) underwent CMT of WOPN. There was no significant difference in the demographic profile of the two groups with biliary pancreatitis being the most frequent cause of acute pancreatitis in both the groups. The mean time until removal of the final percutaneous drain was significantly shorter in the CMT group in comparison to the SPD group (84 days versus 189 days respectively; $p<.006$ ). Three patients in the SPD group developed pancreatic cutaneous fistulae that needed surgery whereas none of the patients in the CMT group developed external pancreatic fistulae or needed surgery. Three patients in the SPD group died during the course of therapy with drainage catheters in place $(2$ because of respiratory failure and 1 of multisystem organ failure) whereas only one patient in the CMT died (because of esophageal carcinoma). Five patients in the SPD group had pseudoaneurysmal bleed whereas none of the patients in the SPD group had pseudoaneurysmal bleed. Patients in the CMT group had significantly decreased length of hospitalization (26 vs. 55 days, $p<.0026$ ), number of computed tomography scans (8.95 vs. $14.3, p<.002)$, and percutaneous drain exchanges $(6.5$ vs. $13, p<.0001)$.

\section{Commentary}

Surgical necrosectomy has been the accepted treatment modality for pancreatic necrosis. However, recently minimally invasive endoscopic and radiological therapeutic modalities have also been shown to be effective in treating WOPN. Endoscopic or radiological drainage alone has been shown to be safe and effective in treating patients with WOPN. However, these techniques are time consuming and require long hospitalization periods thus putting strain on limited hospital resources. Along with this, percutaneous drainage alone is associated with risks and complications like external pancreatic fistulae as well as repeated exposure to ionizing radiations. The authors of the current study have demonstrated that by combining the two techniques ie percutaneous drainage with endoscopic transmural drainage 
the complications, hospital stay and number of radiological procedures done is significantly reduced when compared to percutaneous drainage alone. These results are encouraging and very important for those centre's that have been treating WOPN by the percutaneous route alone. Addition of transmural drainage to standard percutaneous drainage in this study eliminated the risk of development of external pancreatic fistulae as well as surprisingly reduced the incidence of pseudoaneurysmal bleed, possibly because of better pancreatic drainage in the combined group. Although an interesting study, the retrospective nature is a limitation of this study and these results need to be confirmed in multicenter, prospective randomized trials. We also need to look at the long term follow up of these patients, especially the ones with the disconnected pancreatic duct. Moreover, studies are also needed that compare this combined treatment protocol with surgical or endoscopic necrosectomy alone. It will be very interesting to find out what additional advantage the percutaneous drainage will have over and above endoscopic drainage alone.

\section{Large Balloon Dilation vs Mechanical Lithotripsy for the Management of Large Bile Duct Stones: A Prospective Randomized Study}

\author{
G. Stefanidis, N. Viazis, D. Pleskow, \\ S. Manolakopoulos, L. Theocharis, \\ C. Christodoulou, N. Kotsikoros, J. Giannousis, \\ S. Sgouros, M. Rodias, A. Katsikani, R. Chuttani
}

Am J Gastroenterol early online 2 Nov 2010 doi: 10.1038/ ajg. 2010.421

Endoscopic common bile duct (CBD) stone removal is difficult in patients with large stones $(>12 \mathrm{~mm})$, multiple stones, barrel-shaped stones, and tapering course of CBD. Traditionally these patients have been either treated surgically or endoscopic removal of these stones has been attempted after mechanical lithotripsy (ML). Advent of endoscopic large balloon dilatation (LBD) of the papilla following endoscopic sphincterotomy (EST) has offered an alternative for removal of difficult CBD stones. However, the initial enthusiasm with this technique of LBD dampened because of reports of increased risk of pancreatitis. [3] Despite this risk of pancreatitis, LBD is an effective alternative for management of patients with difficult CBD stones. The authors of the current study therefore conducted a prospective randomized controlled trial to compare the therapeutic benefits and complications of EST followed by LBD or ML for the management of large bile duct stones.

A total of 90 patients with large bile duct stones (12 - 20 $\mathrm{mm})$ were randomized to EST followed by LBD $(n=45)$ or EST followed by ML $(n=45)$ [no significant difference in the stone size as well as the CBD diameter between the two groups]. Randomization was performed using the sealed envelope technique and all the ERCP's were performed by a single operator. Patients needing precut for CBD access, with stones more than $20 \mathrm{~mm}$ or with accidental pancreatogram were excluded from the study. In both the groups, complete EST was accomplished by extending the cut up to the major horizontal fold crossing the intramural portion of the bile duct. The patients in the LBD group underwent balloon dilatation with CRE Esophageal / Pyloric balloon, maximum diameter 15, 18, or $20 \mathrm{~mm}$; length $5 \mathrm{~cm}$. (Boston Scientific, Natick, MA). The size of the balloon used was chosen according to the diameter of the bile duct stones and care was taken that it should not exceed the maximum diameter of the bile duct. The balloons were gradually inflated till the waist was obliterated and after that the balloons remain inflated for 10 to 12 seconds. The mechanical lithotripsy was done using a mechanical lithotriptor (BML 4Q, Lithocrush 201 or 202Q; Olympus Optical).

Out of 45 patients in the LBD group, complete CBD clearance was possible in 44 patients $(97.7 \%)$ whereas complete CBD clearance was possible in 41 out of $45(91.1 \%)$ patients in ML group and this difference was not statistically significant. A subgroup analysis, taking into account the bile duct stone size also did not reveal a statistical significant difference in the success rate between the two groups. Presence of a very tortuous CBD resulted in failure of removal of CBD stones in the LBD group whereas failure in ML group was due to basket being broken on a hard stone (1 patient) or inability to capture the stone (3 patients). Significantly more number of complications were noted in the ML group in comparison to the LBD group (9 vs. 2 patients; $p=0.049$ ). One patient in each group developed post-ERCP pancreatitis which was mild and self-limiting and was managed conservatively. Six patients in the ML group developed cholangitis whereas none in the LBD group developed cholangitis $(p=0.026)$. Post ERCP bleeding was noted in one patient in each group. One patient in ML group developed CBD perforation whereas none of the patients in LBD group developed it.

\section{Commentary}

The authors of the current study have shown that EST followed by LBD is equally effective as EST followed by ML for the removal of large bile duct stones, but is associated with fewer complications. This is a prospective randomized study that has shown that LBD is safe and effective and the risk of pancreatitis is low (a feared side effect that has limited its use). The authors believe that the low risk of pancreatitis in their study could be because of the expertise of the endoscopists performing the ERCPs, as well as by the fact that the authors excluded patients at increased risk of pancreatitis ( $>5$ accidental pancreatograms or use of,needle knife precutting). 